\title{
Development and characterization of xenograft model systems for adenoid cystic carcinoma
}

\author{
Christopher A Moskaluk, Alexander S Baras, Stefani A Mancuso, Hao Fan, Robert J Davidson, Dawn C Dirks, \\ Wendy $L$ Golden and Henry F Frierson Jr
}

Adenoid cystic carcinoma (ACC) is one of the most common malignancies to arise in human salivary glands, and it also arises in the glandular tissue of other organ systems. To address the paucity of experimental model systems for this tumor type, we have undertaken a program of transplanting tissue samples of human ACC into immunodeficient nu/nu mice to create xenograft model systems. In 17 of 23 attempts (74\%), xenograft tumors were successfully grown. In all cases, the histologic appearance of the donating tumor was recapitulated in the subsequent xenograft. Characterization of a subset of xenograft models by immunohistochemical biomarkers and by RNA transcript microarray analysis showed good fidelity in the recapitulation of gene expression patterns in the xenograft tumors compared with the human donor tumors. As ACC is known to frequently contain a $t(6 ; 9)$ translocation that fuses the MYB and NFIB genes, fluorescence in situ hybridization (FISH) of 12 ACC xenograft models was performed that assayed MYB locus break-apart and MYB-NFIB locus fusion. Of 12 xenograft models, 11 (92\%) revealed MYB locus rearrangement and 10 (83\%) showed evidence of fusion of the MYB and NFIB loci. The two related xenograft models (derived from primary and metastatic tumors, respectively, of the same human subject) were karyotyped, showing a $t(1 ; 6)$ translocation, suggesting MYB translocation to a novel fusion partner gene. Overall, our results indicate that ACC is amenable to xenografting and that ACC xenograft models recapitulate the molecular and morphologic characteristics of human tumors, suggesting utility as valid experimental and preclinical model systems for this disease.

Laboratory Investigation (2011) 91, 1480-1490; doi:10.1038/labinvest.2011.105; published online 27 June 2011

KEYWORDS: adenoid cystic carcinoma; MYB; NFIB; oncogene fusion; xenograft

Adenoid cystic carcinoma (ACC) is one of the most common malignancies that arise in the salivary glands, ${ }^{1-4}$ with an incidence of 4.5 per $1000000 .^{5}$ It can also develop in glandular tissue closely related to salivary glands such as the lacrimal gland, sinonasal passages, and tracheobronchial tree, as well as in glands of the breast, skin, and vulva. ${ }^{6-8}$ At all of these sites, it is characterized by a distinctive histology of basaloid epithelial cells arranged in cribriform, tubular, or solid patterns, usually demonstrating abundant hyaline extracellular matrix secretion and some degree of myoepithelial differentiation. ${ }^{1}$ ACC is generally a slow-growing tumor characterized by a protracted clinical course, usually well over 5 years in duration, marked by regional recurrence, distant metastasis, and/or spread along peripheral nerves of the head and neck. ${ }^{1,9,10}$ A recurrent chromosomal translocation, $\mathrm{t}(6 ; 9)(\mathrm{q} 23 ; \mathrm{p} 21)$, has been identified in ACCs, ${ }^{11}$ and recently it has been discovered that in a majority of these tumors the $M Y B$ gene on chromosome 6 is fused to the $3^{\prime}$ terminus of the NFIB gene on chromosome 9, creating a fusion gene product resulting in increased $M Y B$-related transcriptional activation. ${ }^{12}$

It has been determined that stable cell lines with putative attribution of ACC derivation are either contaminants of other cell lines or do not have the characteristic MYB-NFIB translocation. ${ }^{13}$ Also, there are no animal models of this histologically and genetically defined tumor type. To address the paucity of experimental and preclinical model systems of ACC, we have been establishing xenograft tumors from clinical samples of ACC, with the hypothesis that such tumors will recapitulate morphologic, genetic, and gene expression signals of the human disease. We describe herein our experience with these models and their characterization.

Department of Pathology, University of Virginia, Charlottesville, VA, USA

Correspondence: Dr CA Moskaluk, MD, PhD, Department of Pathology, University of Virginia, P.O. Box 800214, UVA Health System, Charlottesville, VA 22908, USA. E-mail: cam5p@virginia.edu 


\section{MATERIALS AND METHODS}

\section{Human Subjects and Tissue Procurement}

Fresh tissue samples of ACCs were obtained from remnant tissue of surgical resection specimens performed in the clinical care of patients. Samples were obtained either with informed consent or by anonymous donation from the University of Virginia (UVA) Hospital, through subject-directed donations through the Adenoid Cystic Carcinoma Registry or through the Cooperative Human Tissue Network, with Institutional Review Board approval. Soon after removal from patients, tissues were minced into pieces 1 to $2 \mathrm{~mm}$ in diameter and placed in cell culture media (RPMI) on wet ice for transport to the animal colony. Tissues obtained outside of UVA were sent in culture media on wet ice by overnight courier. For the three xenograft models established from donors outside of the UVA medical system (ACCX14, ACCX15, and ACCX22), the donations were anonymous and formalin-fixed paraffin-embedded tissues were not obtained for comparison.

\section{Tumor Xenografting}

All xenografts reported here were implanted in mice within $36 \mathrm{~h}$ of tissue being removed from the human subjects. Nude mice $(\mathrm{nu} / \mathrm{nu})$ were anesthetized with intraperitoneal injection of Ketamine/Xylazine $60-80 / 5-10 \mathrm{mg} / \mathrm{kg}$. Cutaneous incisions were made in the midscapular and bilateral flank regions, with a subcutaneous pocket created by blunt dissection. One to four fragments of minced tumor ( 1 to $2 \mathrm{~mm}$ in diameter) were placed in each pocket, with subsequent closure of the incision with surgical staples. Tumors were allowed to grow to a maximum diameter of $1-1.5 \mathrm{~cm}$, and were then surgically harvested. Aliquots of the xenograft tumor were minced and passaged into new mice as described above. Initially, the xenografting protocol incorporated the use of Matrigel (BD Biosciences), which was used to soak the tissue fragments before implementation. The use of Matrigel was discontinued, and was not used in the establishment or passage of ACCX11 and subsequent xenograft models.

\section{Histology and Immunohistochemistry}

Immediately upon harvesting from animals, xenograft tumors were sectioned, with a 2- to 3-mm-thick sections placed in neutral buffered formalin for 12-24h. Fixed tissue was then subjected to alcohol dehydration, xylene infusion, and molten paraffin infusion on an automated processor, followed by paraffin embedding. Immunohistochemistry was performed as previously described. ${ }^{14}$ A mouse monoclonal antibody for p63 (catalog no. P3737, Sigma-Aldrich) was used in a 1:1000 dilution. A mouse monoclonal antibody to human large proteoglycan (versican) (clone no. 2-B-1; cata$\log$ no. 270428-1; Associates of Cape Cod) was used in a 1:4000 dilution after microwave antigen retrieval.

\section{RNA Isolation and Gene Expression Profiling}

Frozen tissue samples of ACC xenografts and corresponding human cancers were embedded in OCT compound
(Sakura Finetek) and subjected to cryostat sectioning. The passage number for the xenograft models was as follows: ACCX2, passage 3; ACCX4, passage 1; ACCX6, passage 3; ACCX9, passage 4; ACCX 11, passage 1; and ACCX16, passage 1 . The stained frozen sections were used to direct the dissection of tumor that corresponded to $>80 \%$ tumor cellularity. These dissected subsamples were disrupted using a tissue homogenizer (Pro-200, ProScientific) in lysis buffer supplied in the RNeasy kit (Qiagen) with subsequent RNA isolation using columns and materials supplied by the manufacturer. RNA probe labeling GeneChip ${ }^{\mathrm{TM}}$ hybridization (HG-U133 Plus 2.0 or HG-U133A Affymetrix) were performed in a core research facility (Biomolecular Research Facility at The University of Virginia) using materials and protocols supplied by the manufacturer. The data were processed through the Affymetrix Power Tools suite using quantile normalization, perfect-match $v s$ mismatch correction, and the PLIER algorithm. Statistical significance of differential expression in ACC when compared with normal salivary gland (NSG) tissue was assessed by Wilcoxon's ranksum test with false discovery rate correction. Four common ACC tissue specimens across the two platforms (HG-U133 Plus 2.0 and HG-U133) were used to scale the NSG data for comparison with the paired ACC and ACC xenograft data assayed on the HG-U133 Plus 2.0 platform. The expression data of a given probe set were normalized using a weighted $z$-score approach. Subsequently, hierarchical clustering was conducted using the furthest neighbor algorithm (complete linkage) with the Euclidean distance metric. Files containing the raw probe hybridization data have been deposited at the Gene Expression Omnibus data repository (http:// www.ncbi.nlm.nih.gov/geo/) with series accession number GSE28996.

\section{Tissue Microarray (TMA) Manufacture}

Two TMAs were constructed for these studies. One consisted of matched samples of xenograft tumors and the human tumors from which the xenografts were derived, for those models in which the human tumors were available. This TMA was constructed with three $0.6 \mathrm{~mm}$ diameter cores sampling each specimen on a MTA-1 TMA apparatus (Beecher). The second TMA, used for the fluorescence in situ hybridization (FISH) analysis, consisted entirely of xenograft samples and included all of the models described here. This TMA was constructed with three $1.5 \mathrm{~mm}$ diameter cores sampling each specimen, constructed with a TMArrayer apparatus (Pathology Devices).

\section{Fluorescence In Situ Hybridization}

Nick translated probes produced from bacterial artificial chromosome (BAC) clones were obtained from Empire Genomics as a hybridization mixture with human cot-1 DNA. TMA slides were incubated at $56^{\circ} \mathrm{C}$ overnight, deparaffinized using xylene, and rehydrated using a graded series of ethanol baths. Slides were heated in target retrieval 
solution (Dako) at $95^{\circ} \mathrm{C}$ for $40 \mathrm{~min}$, rinsed with distilled water for $5 \mathrm{~min}$, digested with Proteinase $\mathrm{K}$ at room temperature for $8 \mathrm{~min}$, rinsed with distilled water for $5 \mathrm{~min}$, and then dehydrated with a graded series of alcohol baths. For the MYB break-apart FISH assay, BAC clone RP11-104D9 labeled with 5-Fluorescein and BAC clone RP11-170P19 labeled with 5-carboxy-X-rhodamine (5-Rox) were used. For the MYBNFIB fusion FISH, BAC clone RP11-104D9 labeled with 5-Fluorescein and BAC clone RP11-452J2 labeled with 5-Rox were used. The probe/in situ hybridization buffer mixture was distributed across the microarray area, covered with a glass cover slip, and sealed with rubber cement. Slides were incubated in a humidified chamber at $73^{\circ} \mathrm{C}$ for $5 \mathrm{~min}$ to denature the microarray DNA followed by $37^{\circ} \mathrm{C}$ for $18 \mathrm{~h}$ to hybridize with the probes. After hybridization, slides were washed with $2 \times \mathrm{SSC}$ at room temperature for $5 \mathrm{~min}$, $0.4 \times \mathrm{SSC} / 0.3 \% \mathrm{NP}-40$ at $73{ }^{\circ} \mathrm{C}$ for $2 \mathrm{~min}, 2 \times \mathrm{SSC} / 0.1 \%$ $\mathrm{NP}-40$ at room temperature for $1 \mathrm{~min}$, and rinsed with distilled water at room temperature for $1 \mathrm{~min}$. Slides were counterstained using DAPI in Vectashield mounting medium (Vector Laboratories), covered with a glass cover slip, and sealed with CytoSeal XYL. Slides were examined on a fluorescence microscope (Imager A.2, Carl Zeiss Imaging Solutions $\mathrm{GmbH}$ ) equipped with a $\times 150$ Plan-APOCHROMA objective and images captured using a digital camera (AxioCam MRm, Zeiss) controlled by AxioVision software (Release 4.8, Zeiss). A minimum of 100 nuclei were examined per specimen to obtain the consensus probe count.

\section{Karyotype Analysis}

The related ACCX2 and ACCX6 xenograft model systems (primary tumor and metastatic tumor from same patient, respectively) were subjected to karyotype analysis. Culturing, harvesting, and GTG banding of the xenograft samples were performed using standard techniques. ${ }^{15}$ Xenograft tissues were aseptically resected from mice and minced. Minced tissue was divided and half was resuspended in $1 \mathrm{ml}$ complete minimum essential medium (MEM, Gibco) plus 20\% fetal bovine serum (Gibco), 1\% penicillin/streptomycin solution, and $1 \% \mathrm{~L}$-glutamine. Approximately $0.5 \mathrm{ml}$ of cell suspension was inoculated onto each of four sterile $22 \mathrm{~mm}$ cover slips placed in sterile $35 \mathrm{~mm}$ tissue culture dishes. The remaining minced tissue was placed into a sterile polypropylene $15 \mathrm{ml}$ conical tube and centrifuged at $140 \mathrm{~g}$ for $10 \mathrm{~min}$. The medium was aspirated and replaced with $3 \mathrm{ml}$ Collagenase Type IV-S (Sigma) rehydrated in incomplete MEM at a final concentration of $1 \mathrm{mg} / \mathrm{ml}$. The tissue was incubated at $37^{\circ} \mathrm{C}$ for $\sim 1 \mathrm{~h}$, or until tissue appeared completely digested upon visual inspection. The digested tissue was washed two times in complete MEM, resuspended in $1 \mathrm{ml}$ complete MEM, and plated as described above. All dishes were incubated at $37^{\circ} \mathrm{C}$ and $5 \% \mathrm{CO}_{2}$ until harvest. Cover slips were flooded after $24 \mathrm{~h}$ with $1 \mathrm{ml}$ complete MEM and cultured for $48-72 \mathrm{~h}$.

Cover slips were harvested using standard techniques that include arresting the cells in metaphase with overnight colcemid $(200 \mu \mathrm{g} / \mathrm{ml}$, Gibco), $2 \mathrm{ml} 4 \%$ (w/v) potassium chloride hypotonic treatment, and stabilization with modified Carnoy's fixative (1:3 glacial acetic acid/methanol). Cover slips were air dried in a humidified chamber adjusted to $\sim 45 \%$ humidity and $78{ }^{\circ} \mathrm{C}$ and incubated on a $70{ }^{\circ} \mathrm{C}$ slide warmer for $\sim 3 \mathrm{~h}$ before staining. $\mathrm{G}$ banding after treatment with trypsin and Giemsa staining (GTG) of the cover slips was performed as follows: cover slips were digested for $\sim 25 \mathrm{~s}$ in trypsin (Difco) diluted in Hank's balanced salt solution (Gibco) to a final concentration of $0.2 \mu \mathrm{g} / \mathrm{ml}$. Cover slips were rinsed $2 \times$ in $0.9 \% \mathrm{NaCl}$, stained for $2 \mathrm{~min}$ in Giemsa stain (VWR) diluted in Gurr's Buffer (VWR) at a final concentration of $4 \%(\mathrm{v} / \mathrm{v})$ Giemsa, dried with bibulous paper, and mounted to slides for karyotypic analysis. Metaphase cells were examined using brightfield microscopy (Olympus). Human metaphase cells were analyzed for chromosome number and structure. Karyotypes were prepared and described with ISCN nomenclature. ${ }^{16}$

\section{RESULTS}

\section{Establishment of ACC Xenograft Models}

Subcutaneous xenografting of fresh ACC tumor samples, within a 36-h time period of removal from the patient, was attempted 23 times. In 17 of these attempts (74\%), xenografted tumors grew to a size $\geq 1 \mathrm{~cm}$ in maximum dimension, and were histologically confirmed as tumors with features characteristic of ACC. Table 1 shows the characteristics of cases that were successfully xenografted and Table 2 shows the characteristics of cases in which xenografts did not grow. There were no statistically significant differences for tumors that were successfully grown after implantation and those that did not when examining donor age, donor gender, tumor site of origin, or tumor grade. Of note, in 2007, it was discovered that the commercial Matrigel preparations that were being used in the xenografting protocol were contaminated with lactate dehydrogenase elevating virus (LDEV), causing a wasting syndrome in some animals in the xenograft colony. The use of Matrigel was discontinued at this time after xenograft ACCX11, with no apparent diminishment of tumor engraftment. Of the 17 models described here, 15 were successfully transferred at least once to subsequent mice. At the time of preparation of this manuscript, 12 of the models are actively being serially passaged between mice, with the number of passages indicated in Table 1.

\section{Histologic Characterization}

All the ACC-xenografted tumors were examined histologically, either after formalin fixation and paraffin embedding or by frozen tissue sectioning. The majority of tumors arising in xenografted mice showed the characteristic histology of the donor tumor (representative images are shown in Figure 1). Three donor tumors did not have FFPE tissue samples available for comparison (ACCX14, ACCX15, and ACCX22), but the histologic features of these xenografts were also consistent with ACC. There were several instances of spon- 
Table 1 Successful ACC xenograft cases

\begin{tabular}{|c|c|c|c|c|c|c|c|c|}
\hline \multirow{2}{*}{$\begin{array}{l}\text { Xenograft } \\
\text { designation }\end{array}$} & \multicolumn{3}{|c|}{ Donor information } & \multicolumn{3}{|c|}{ Tumor information } & \multirow{2}{*}{$\begin{array}{l}\text { Time to } \\
\text { first harvest } \\
\text { (months) }\end{array}$} & \multirow{2}{*}{$\begin{array}{c}\text { Last } \\
\text { passage }\end{array}$} \\
\hline & $\begin{array}{c}\text { Donor } \\
\text { designation }\end{array}$ & Age & Sex & Status & Site & Grade & & \\
\hline ACCX2002.1 & 1 & 26 & M & Primary tumor & Parotid gland & 2 & 12 & $1^{\mathrm{b}}$ \\
\hline ACCX1 & 3 & 53 & M & Primary tumor & Sinonasal cavity & 1 & 16 & $1^{\mathrm{b}}$ \\
\hline ACCX2 & 4 & 33 & M & Primary tumor & Parotid gland & 2 & 5 & 8 \\
\hline ACCX6 & 4 & \multicolumn{3}{|c|}{ Same pt. as ACCX2, metastasis obtained 1 year later } & Metastatic tumor to lungs & 2 & 4 & 12 \\
\hline ACCX4 & 5 & 46 & $\mathrm{~F}$ & Primary tumor & Oral cavity & 2 & 8 & $2^{b}$ \\
\hline ACCX11 & 8 & 55 & $\mathrm{~F}$ & Primary tumor & Sinonasal cavity & 3 & 10 & 5 \\
\hline ACCX12 & 9 & 16 & $\mathrm{~F}$ & Primary tumor & Trachea & 2 & 5.5 & 6 \\
\hline ACCX14 & 10 & 40 & $\mathrm{~F}$ & Primary tumor & Trachea & 1 & 5 & 6 \\
\hline ACCX15 & 11 & 58 & M & Primary tumor & Oral cavity & Unknown & 11 & 4 \\
\hline ACCX16 & 12 & 40 & $\mathrm{~F}$ & Primary tumor & Bronchus & 2 & 6 & 3 \\
\hline ACCX19 & 13 & 49 & $\mathrm{~F}$ & Primary tumor & Oral cavity & 2 & 5 & 3 \\
\hline ACCX22 & 14 & 36 & $\mathrm{~F}$ & Primary tumor & Parotid gland & 1 & 6 & 3 \\
\hline
\end{tabular}

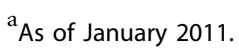

${ }^{\mathrm{b}}$ Not being actively passaged at the time of publication.

Table 2 Unsuccessful ACC xenograft attempts

\begin{tabular}{|c|c|c|c|c|c|}
\hline \multirow[t]{2}{*}{ Designation } & \multicolumn{3}{|c|}{ Donor information } & \multicolumn{2}{|c|}{ Tumor information } \\
\hline & Age & Gender & Status & Site & Grade \\
\hline ACCX2003.1 & 55 & M & Primary tumor & Sinonasal cavity & 2 \\
\hline ACCX2004.1 & 51 & M & Primary tumor & Sinonasal cavity & 1 \\
\hline ACCX7 & 42 & M & Primary tumor & Oral cavity & 2 \\
\hline ACCX8 & 43 & M & Recurrent tumor & Oral cavity & 3 \\
\hline ACCX10 & 56 & $\mathrm{~F}$ & Primary tumor & Bronchus & 1 \\
\hline ACCX17 & 47 & $\mathrm{~F}$ & Primary tumor & Oral cavity & 2 \\
\hline
\end{tabular}

taneous development of murine tumors in the xenograft animal colony: two squamous carcinomas and four lymphomas. These tumors were identified as contaminating artifacts of the xenografting process by microscopic examination of histologic quality control tissue preparations and by immunohistochemical characterization that was consistent with murine tissue (data not shown).

Because of the relatively slow growth of ACC, most of the xenograft model systems described here are of low passage number $(<10)$. For the few models with greater passage numbers, the histologic features of the model have been retained in the late passages (data not shown).

\section{Immunohistochemical Characterization}

The transcription factor p63 has been shown to be expressed in a subset of tumor nuclei in the majority of ACCs. ${ }^{17,18}$ Additionally, we have previously identified a characteristic gene expression profile of ACC using microarrays ${ }^{14}$ that had shown an elevated expression of a variety of extracellular matrix gene products, consistent with morphologic characteristics of this tumor type. Among these was versican, for which we used immunohistochemistry for detecting protein expression. These immunohistochemical stains were applied to a TMA containing matched samples of a subset of the ACC xenograft models and the human cancers from which they were derived. In all cases, the expression pattern of the biomarker cohort was similar between donor tumors and their xenografted derivatives. Representative examples of IHC are shown in Figure 1.

\section{Transcript Gene Expression Profiling}

In a broader survey of gene expression profiling, we analyzed samples of primary tumors, NSG tissue, and xenografted 


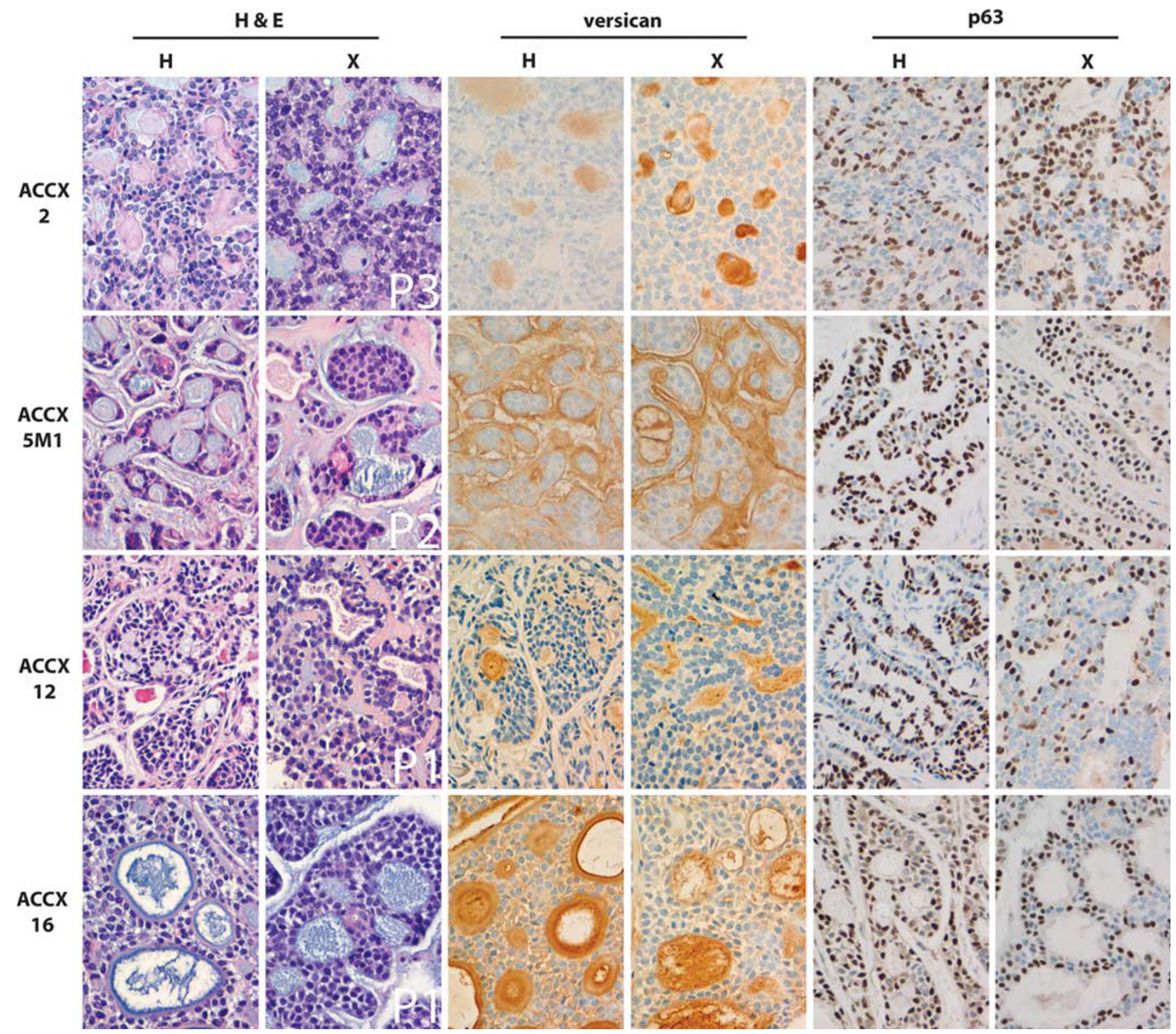

Figure 1 Histology and immunohistochemistry of human tumors and derivative xenograft tumors. Hematoxylin and eosin (H\&E)-stained histologic images of human $(\mathrm{H})$ ACC tumors and their xenografts $(\mathrm{X})$. The passage number $(\mathrm{P})$ of the xenograft tumor being depicted is noted in the image. Similarly, representative images of immunohistochemistry $(\mathrm{IHC})$ for versican and p63 are shown for the paired samples. In all instances, the xenograft tumors recapitulated the histologic and protein expression patterns of the human tumors from which they were derived. The IHC was performed on tissue from the same passage number xenograft as depicted in the H\&E images (original magnification $\times 400$ ).

tumors by oligonucleotide microarrays (HG-U133 plus 2.0 and HG-U133A, Affymetrix). From differential expression analysis between primary human ACC and NSG tissue samples on the HG-U133A platform, 1271 probe sets were identified as differentially expressed (false discovery rate $<0.05$ and minimum absolute difference $>100$ ). Using this subset of differentially expressed genes in unsupervised clustering analysis of the xenograft tumors and human ACC samples, the result showed that in almost all circumstances the xenografted tumors clustered most closely to the human tumor sample from which they were derived (Figure 2). The only exception was in the case where a primary human tumor clustered most closely to a sample of a subsequent metastasis from the same subject (donor no. 4). Notably, the xenograft models derived from these primary (ACCX2) and metastatic (ACCX6) tumor samples clustered closest to each other, and formed a separate clade with the human tumors from where they were derived. Additionally, the xenografted tumors showed conserved differential expression when compared with NSG samples. Overall, this analysis indicates that the xenograft tumors maintain durable gene expression profiles that closely resemble the human tumors from which they were derived, and that the foreign murine host milieu does not cause gene expression differences so profound that the xenografted tumors cluster away from their derivatives. 


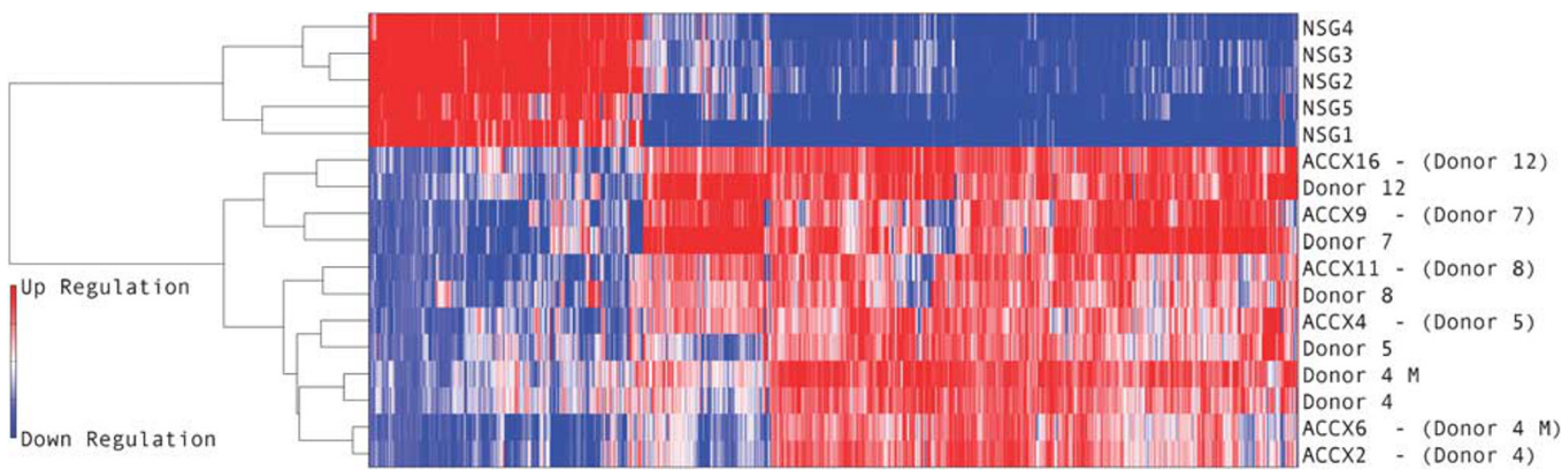

Figure 2 Clustering of gene expression profiling data. A subset of xenograft tumors and samples of the corresponding human tumor from which they were derived were subjected to gene expression profiling on HU133 Plus 2.0 GeneChips. Shown here is a heat map of the 1271 probe sets that distinguish ACC tumors from normal salivary gland (NSG) tissue, and to the left of the heat map, the graphical results of unsupervised clustering analysis of the expression profiles, using the furthest neighbor algorithm (complete linkage) with the Euclidean distance metric. The human tumors and the xenograft tumors cluster together and away from the NSG samples, with clear populations of differentially regulated genes between the malignant and benign tissue samples. In almost every case, the xenograft tumor clusters most closely with the human tumor from which it was derived. The only exceptions were ACCX2 and 6, which were derived from primary and metastatic tumors from the same patient, in which the human samples and xenografts clustered closer to each other than to their related xenograft.

Table 3 Results of MYB break-apart FISH

\begin{tabular}{|c|c|c|c|c|c|}
\hline $\begin{array}{l}\text { Xenograft } \\
\text { model }\end{array}$ & $\begin{array}{l}\text { Passage } \\
\text { no. } \\
\text { tested }\end{array}$ & $\begin{array}{l}\text { No. of } \\
\text { intact } \\
\text { probe pairs }\end{array}$ & $\begin{array}{c}\text { No. of } \\
\text { separate } \\
\text { green signals } \\
\text { (centromeric } \\
\text { probe) }\end{array}$ & $\begin{array}{l}\text { No. of separate } \\
\text { red signals } \\
\text { (telomeric } \\
\text { probe) }\end{array}$ & Interpretation \\
\hline $\mathrm{ACC} \times 2$ & 3 & 2 & 1 & 0 & $\begin{array}{l}\text { Rearrangement of } 1 M Y B \text { locus with loss of } 6 p \text {. Duplication of second intact } M Y B \\
\text { locus }\end{array}$ \\
\hline $\mathrm{ACC} \times 5 \mathrm{M} 1$ & 4 & 1 & 1 & 1 & Rearrangement of $1 M Y B$ locus. Retention of one intact $M Y B$ locus \\
\hline $\mathrm{ACC} \times 6$ & 4 & 1 & 1 & 0 & Rearrangement of 1 MYB locus with loss of $6 \mathrm{p}$. Retention of one intact MYB locus \\
\hline $\mathrm{ACC} \times 9$ & 4 & 0 & 2 & 1 & Duplication of rearranged MYB locus. Loss of second MYB locus \\
\hline ACCX11 & 2 & 1 & 1 & 0 & Rearrangement of $1 M Y B$ locus with loss of $6 p$. Retention of one intact $M Y B$ locus \\
\hline ACCX12 & 5 & 1 & 2 & 0 & $\begin{array}{l}\text { Duplication of rearranged } M Y B \text { locus, with loss of } 6 \mathrm{p} \text {. Retention of one intact } M Y B \\
\text { locus }\end{array}$ \\
\hline ACCX14 & 2 & 1 & 1 & 1 & Rearrangement of 1 MYB locus. Retention of one intact MYB locus \\
\hline ACCX15 & 2 & 1 & 1 & 0 & Rearrangement of $1 M Y B$ locus with loss of $6 p$. Retention of one intact $M Y B$ locus \\
\hline ACCX16 & 1 & 1 & 1 & 0 & Rearrangement of 1 MYB locus with loss of $6 \mathrm{p}$. Retention of one intact $M Y B$ locus \\
\hline ACCX19 & 1 & 2 & 1 & 0 & $\begin{array}{l}\text { Rearrangement of } 1 M Y B \text { locus with loss of } 6 \mathrm{p} \text {. Duplication of second intact } M Y B \\
\text { locus }\end{array}$ \\
\hline ACCX2 M1 & 1 & 1 & 1 & 1 & Rearrangement of 1 MYB locus. Retention of one intact MYB locus \\
\hline ACCX22 & 1 & 2 & 0 & 0 & Wild-type configuration (2 intact MYB loci) \\
\hline
\end{tabular}

\section{MYB Locus Break-Apart FISH Analysis}

The 12 xenograft cases that were actively being passaged were examined for the integrity of the MYB locus using the centromeric BAC probe RP11-104D9, labeled green with fluorescein and the telomeric BAC probe RP11-170P19 labeled red with 5-ROX. Of 12 xenograft models, 11 (92\%) showed separation of one of the red/green probe pairs, or specific loss of the telomeric probe, consistent with rearrangement of the MYB gene locus (Table 3). Figure 3 shows representative examples of $M Y B$ break-apart FISH assays.

FFPE tissue samples of the human tumors from which the xenografts were derived were available for 9 of the 12 


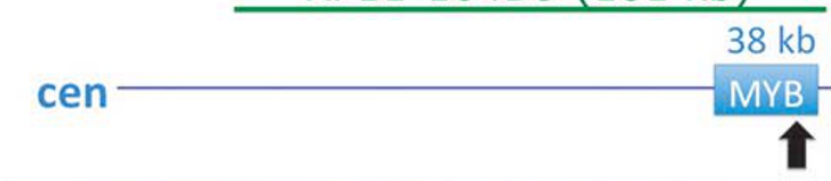

b
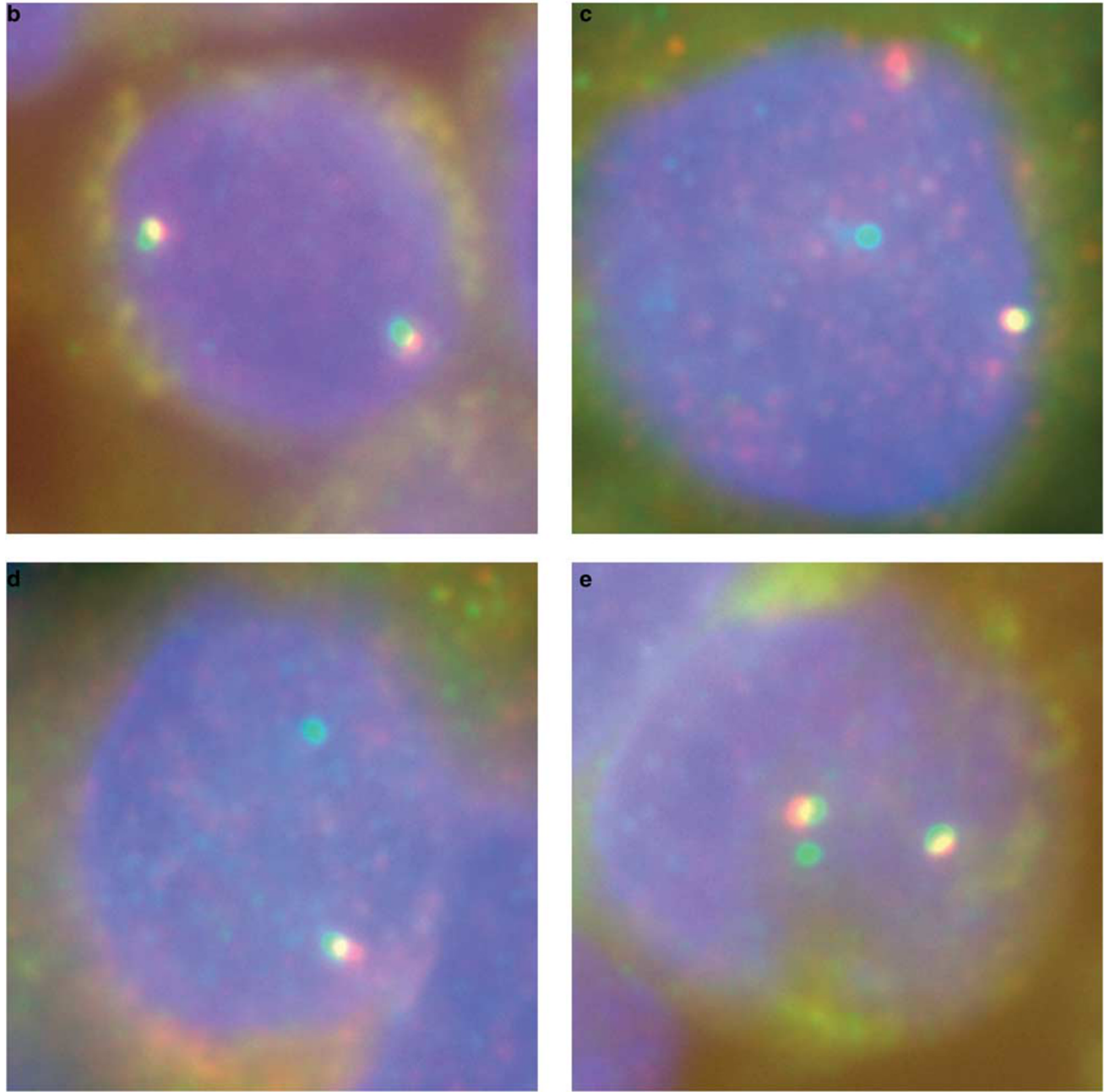

Figure 3 Examples of MYB break-apart FISH. (a) The 38-kilobase (kb) MYB gene locus is shown schematically, oriented to the centromeric (cen) and telomeric (tel) aspect of chromosome 6 . The characteristic location of the breakpoint involved in the $t(6 ; 9)$ translocation is shown by a black arrow. The location of the BAC clones used as FISH probes are shown above the locus (drawn to scale) and colored to match the fluor used in the FISH assays. (b) ACCX22 displaying two red/green/yellow signals indicating two intact MYB loci. (c) ACCX14 displaying one red/green/yellow signal, one red signal, and one green signal consistent with a MYB locus rearrangement. (d) ACCX16 displaying one red/green/yellow signal and one green signal consistent with a MYB locus rearrangement. (e) ACCX19 displaying two red/green/yellow signals and one green signal consistent with a rearrangement of one MYB locus and a duplication of the wild-type $M Y B$ locus. 
xenograft models (all except ACCX14, ACCX15, and ACCX22). These tissues were also examined with the BAC probes as described above. All nine human donor tumors showed the same MYB locus status as their derivative xenografts, ruling out the rearrangement occurring as a consequence of xenografting.

\section{MYB-NFIB Fusion FISH Analysis}

The same 12 xenograft cases described above were examined for the translocation of the NFIB locus on chromosome 9 to the MYB locus on chromosome 6, using the BAC probe centromeric to the MYB locus, RP11-104D9, labeled green with fluorescein and the BAC probe telomeric to the NFIB locus, RP11-170P19 labeled red with 5-ROX. Of the 12 xenograft models, $10(83 \%)$ displayed a pairing of red and green signals, consistent with a MYB-NFIB gene fusion event (Table 4). Figure 4 shows representative examples of $M Y B-$ NFIB fusion FISH assays. The two xenograft models that did not show evidence of the $t(6 ; 9)$ translocation were related: ACCX2 and ACCX6, derived from the primary tumor and a metastatic tumor from the same patient. The fact that these xenografts had demonstrated findings in the break-apart FISH of a $M Y B$ locus rearrangement suggested an alternate chromosomal rearrangement event. There was only one discrepancy noted between the break-apart and fusion FISH analyses, and that was for ACCX22, in which the break-apart FISH showed two intact red/green probe pairs, but the fusion FISH was consistent with a MYB-NFIB locus fusion event.

\section{Karyotype Analysis of ACCX2 and ACCX6}

Given that these related xenograft models appeared to have the MYB gene locus rearranged, but not fused to the char- acteristic NFIB locus on chromosome 9, karyotype analysis was performed to study their chromosomal structure. A total of 15 metaphase cells were available for analysis from ACCX2. The consensus karyotype for this xenograft was 45, $\mathrm{X},-\mathrm{Y}, \mathrm{t}(1 ; 6)(\mathrm{p} 22 ; \mathrm{q} 13)$, inv(5)(q13q33). Only five metaphases were found from ACCX6; however, all had the same karyotype as ACCX2: 45, X,-Y, t(1;6)(p22;q13), inv(5)(q13q33). Representative karyotypes are shown in Figure 5.

\section{DISCUSSION}

ACC is the most common malignancy to arise in the submandibular salivary gland, the lacrimal gland, and the minor salivary glands, and is also one of the most common cancers of the parotid and sublingual salivary glands. ${ }^{2-5,6}$ Typical presenting symptoms for patients include pain and facial nerve paralysis, as ACC has a proclivity for invading nerves, a feature that suggests specific molecular pathways leading to neurotropism. This neurotropism also contributes to the infiltrative nature of this neoplasm, with deep penetration of vital structures of the craniofacial region along major nerve trunks. The 5-year survival rate is relatively good, but the survival rate becomes poor at 10-20 years after treatment. ACC is resistant to radiation therapy and standard chemotherapeutic agents and most patients who develop metastases or inoperable local recurrences die of their disease. Because of these clinical features, ACC has been described as 'one of the most biologically destructive and unpredictable tumors of the head and neck.' ${ }^{19}$

The establishment of appropriate model systems is a critical step in understanding the biology of any disease process. Given the current paucity of validated cell lines, the xenografted tumors described here are potentially very

Table 4 Results of MYB-NFIB fusion FISH

\begin{tabular}{|c|c|c|c|c|c|}
\hline $\begin{array}{l}\text { Xenograft } \\
\text { model }\end{array}$ & $\begin{array}{c}\text { Passage } \\
\text { no. } \\
\text { tested }\end{array}$ & $\begin{array}{l}\text { No. of } \\
\text { fused } \\
\text { probe pairs }\end{array}$ & $\begin{array}{l}\text { No. of separate } \\
\text { green signals } \\
\text { (MYB probe) }\end{array}$ & $\begin{array}{l}\text { No. of separate } \\
\text { red signals } \\
\text { (NFIB probe) }\end{array}$ & Interpretation \\
\hline $\mathrm{ACC} \times 2$ & 3 & 0 & 3 & 2 & NFIB not translocated to $M Y B$ locus. Duplication of one $M Y B$ locus \\
\hline ACCX5M1 & 4 & 1 & 1 & 1 & NFIB translocated to MYB locus \\
\hline $\mathrm{ACCX} 6$ & 4 & 0 & 2 & 2 & NFIB not translocated to $M Y B$ locus \\
\hline $\operatorname{ACCX} 9$ & 4 & 2 & 1 & 1 & NFIB translocated to MYB locus. Duplication of translocation \\
\hline ACCX11 & 2 & 1 & 1 & 1 & NFIB translocated to $M Y B$ locus \\
\hline ACCX12 & 5 & 1 & 2 & 1 & NFIB translocated to MYB locus. Duplication of one MYB locus \\
\hline $\mathrm{ACCX} 14$ & 2 & 1 & 1 & 1 & NFIB translocated to $M Y B$ locus \\
\hline ACCX15 & 2 & 1 & 1 & 1 & NFIB translocated to $M Y B$ locus \\
\hline ACCX16 & 1 & 1 & 1 & 1 & NFIB translocated to $M Y B$ locus \\
\hline ACCX19 & 1 & 1 & 2 & 1 & NFIB translocated to MYB locus. Duplication of one MYB locus \\
\hline $\mathrm{ACC} \times 2 \mathrm{M} 1$ & 1 & 1 & 1 & 1 & NFIB translocated to MYB locus \\
\hline ACCX22 & 1 & 1 & 1 & 1 & NFIB translocated to MYB locus \\
\hline
\end{tabular}



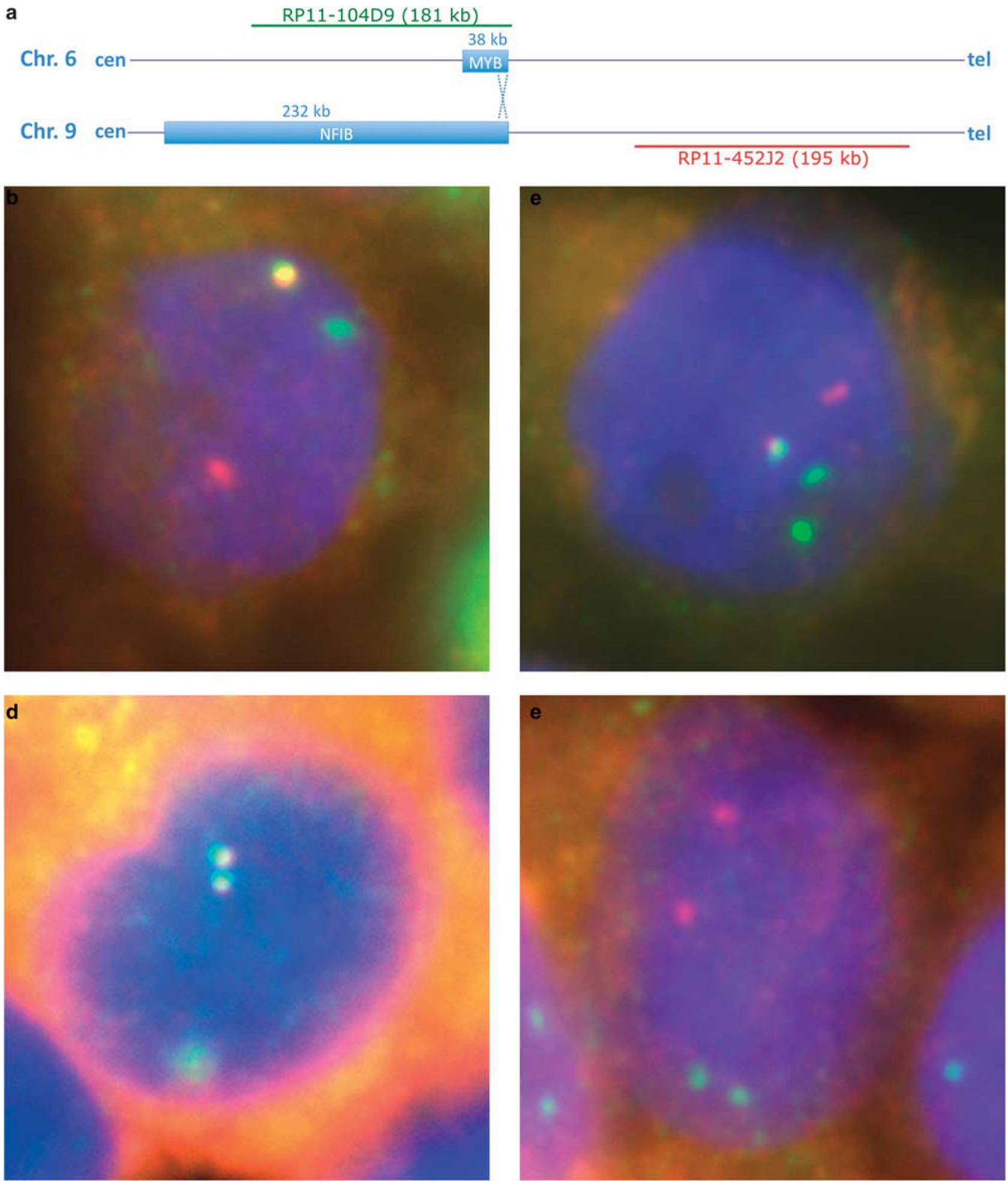

Figure 4 Examples of MYB-NFIB fusion FISH. (a) The MYB gene locus on chromosome 6 and the NFIB gene locus on chromosome 9 are shown schematically, oriented to the centromeric (cen) and telomeric (tel) aspect of their respective chromosomes. The characteristic location of the $t(6 ; 9)$ translocation involved is shown by the crossed dotted lines. The location of the BAC clones used as FISH probes are shown above the MYB locus and below the NFIB locus, (drawn to scale) and colored to match the fluor used in the FISH assays. (b) ACCX22 displaying one yellow signal indicating a fusion of the MYB and NFIB loci. Separate unfused MYB and NFIB signals are also seen. (c) ACCX19 displaying one red/green signal indicating a fusion of the MYB and NFIB loci. Two additional separate green MYB signals are present, indicating duplication on one non-fused MYB locus. A separate red NFIB signal is also seen. (d) ACCX9 displaying two yellow signals consistent with a duplication of fused MYB-NFIB loci. (e) ACCX2 displaying two separate green signals and two separate red signals indicating that the NFIB locus has not been fused to the MYB locus in this tumor. 


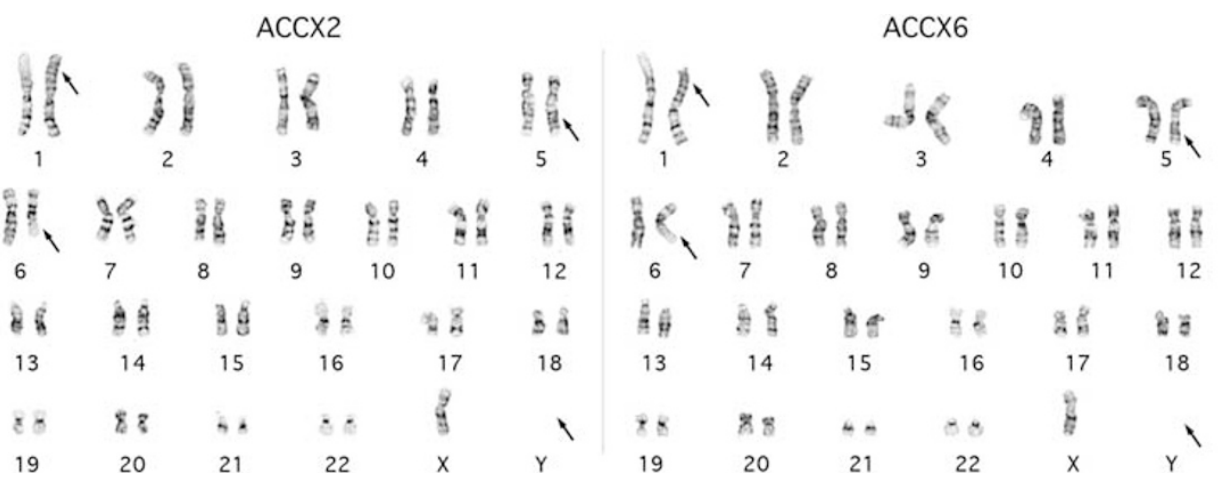

Figure 5 Representative karyotypes of ACCX2 and ACCX6. The karyotype of ACCX2 is shown with arrows demonstrating the translocation between chromosomes 1 and 6, an inverted 5, and loss of the $Y$ chromosome. A representative karyotype of ACCX6 demonstrates the identical abnormalities. The karyotype designation for both $A C C X 2$ and ACCX6 is as follows: 45, $X,-Y, t(1 ; 6)(p 22 ; q 13), \operatorname{inv}(5)(q 13 q 33)$.

important model systems for ACC. We have shown that these xenografted ACCs recapitulate the typical morphologic and gene expression signature of the human disease. Perhaps most importantly, we have determined that all of the models tested have some type of rearrangement involving the $M Y B$ locus, with the majority of models displaying a characteristic $\mathrm{t}(6 ; 9)$ translocation that results in the MYB and NFIB gene fusion. Two recent independent studies suggest that the majority of ACCs contain a MYB-NFIB fusion gene; ${ }^{12,20}$ thus, it is likely that this represents a 'driver mutation' for this tumor type. These xenograft lines may be useful to prove this hypothesis by manipulation using small interfering RNAs or other gene silencing technology. Although the majority of the xenografts appear to contain the MYB-NFIB rearrangement, the ACCX2 and ACCX6 xenografts appear to have an anomalous $\mathrm{t}(1 ; 6)$ translocation, presumably fusing $M Y B$ to a different gene partner. Further study of these models may shed light into common mechanisms of MYB activation by different fusion partners. Further study is also required for one xenograft model, ACCX22, which has discrepant results in the two FISH assays. Although two intact red/green probe pairs were detected in this model for the $M Y B$ break-apart assay, a fusion pair was observed with MYB-NFIB probe pairs. One hypothesis to be explored is whether an insertional event has occurred, bringing a small section of chromosome 9 to the $M Y B$ locus without translocation of the distal portion of chromosome 6. The MYB break-apart FISH assay was repeated for this sample, but it could not be confidently determined if one of the two probe pairs were spaced further apart than normal.

The ACC xenograft tumors showed a faithful recapitulation of the histologic morphology and the gene expression profiles of the parent tumors. Although there were a few differences noted between primary human and xenografted tumor samples, it was remarkable that global gene expression profiles showed that the xenograft tumors were more like the human tumors from which they were derived from, rather than the xenografts clustering together as a group. This analysis suggests that the gene expression profiles unique to a particular tumor survive durably in the xenografting process, and that the altered microenvironment because of the presence of non-human stromal cells does not alter the majority of the tumor cell gene expression signature. It is possible that some of the differences noted between human- and mouse-derived tumor samples may actually be because of the fact that the human samples contain a minor population of non-neoplastic stromal cells that may contribute more to the detectable transcripts by the array probes than the murine stromal cells supporting the xenografted tumors.

Xenograft tumors are highly utilized preclinical model systems to examine experimental therapeutic modalities before human testing. Many if not most xenograft systems employ the formation of tumors from cancer cells adapted to long-term cell culture. Such models have been criticized recently for their lack of correlation to response of therapeutic manipulation by actual human tumors in the clinical setting. ${ }^{21,22}$ This may be explained by phenotypic and genetic drift of tumor cells adapted to cell culture for hundreds if not thousands of passages. However, there has been recent evidence that xenograft models derived directly from human tumors and passaged only as xenografts from animal to animal retain characteristics that make them more representative models of the human disease. ${ }^{23,24}$ We suggest that the xenograft models we have derived and described here may serve as reliable model systems for the testing of chemotherapeutic sensitivity and other therapeutic modalities for ACC.

\section{ACKNOWLEDGEMENTS}

This work was supported by a grant from the National Institute of Dental and Craniofacial Research (NIDCR; no. RC1-DE020687), and by grants from the National Organization of Rare Disorders and the Adenoid Cystic Carcinoma Research Foundation. We thank Alexandra Moskaluk for assistance in tissue microarray construction and Patcharin Pramoonjago and Sharon Birdsall for immunohistochemistry services.

\section{DISCLOSURE/CONFLICT OF INTEREST}

The authors declare no conflict of interest. 
1. Ellis GL, Auclair PL. Tumors of the Salivary Glands, Vol. Fascicle 17, 3rd edn AFIP: Washington, DC, 1996.

2. Spiro RH, Huvos AG, Strong EW. Adenoid cystic carcinoma of salivary gland origin: a clinicopathologic study of 242 cases. Am J Surg 1974;128:512-520.

3. Isacsson G, Shear M. Intraoral salivary gland tumors: a retrospective study of 201 cases. J Oral Pathol 1983;12:57-62.

4. Eveson JW, Cawson RA. Salivary gland tumours. A review of 2410 cases with particular reference to histological types, site, age and sex distribution. J Pathol 1985;146:51-58.

5. Bonaparte JP, Hart R, Trites J, et al. Incidence of adenoid cystic carcinoma in Nova Scotia: 30-year population-based epidemiologic study. J Otolaryngol Head Neck Surg 2008;37:642-648.

6. Wiseman SM, Popat SR, Rigual NR, et al. Adenoid cystic carcinoma of the paranasal sinuses or nasal cavity: a 40 -year review of 35 cases. Ear Nose Throat J 2002:81:510-514, 516-517.

7. Font RL, Smith SL, Bryan RG. Malignant epithelial tumors of the lacrimal gland: a clinicopathologic study of 21 cases. Arch Ophthalmol 1998;116:613-616.

8. Ghabach B, Anderson W, Curtis R, et al. Adenoid cystic carcinoma of the breast in the United States (1977 to 2006): a population-based cohort study. Breast Cancer Res 2010;12:R54.

9. Vander Poorten VL, Balm AJ, Hilgers FJ, et al. Prognostic factors for long term results of the treatment of patients with malignant submandibular gland tumors. Cancer 1999:85:2255-2264.

10. Fordice J, Kershaw C, el-Naggar A, et al. Adenoid cystic carcinoma of the head and neck. Predictors of morbidity and mortality. Arch Otolaryngol Head Neck Surg 1999;125:149-152.

11. Nordkvist A, Mark J, Gustavsson $\mathrm{H}$, et al. Nonrandom chromosome rearrangements in adenoid cystic carcinoma of the salivary glands. Genes Chromosomes Cancer 1994;10:115-121.

12. Persson M, Andren Y, Mark J, et al. Recurrent fusion of MYB and NFIB transcription factor genes in carcinomas of the breast and head and neck. Proc Natl Acad Sci USA 2009;106:18740-18744.

13. Phuchareon J, Ohta Y, Woo JM, et al. Genetic profiling reveals cross-contamination and misidentification of 6 adenoid cystic carcinoma cell lines: ACC2, ACC3, ACCM, ACCNS, ACCS and CAC2. PLOS ONE 2009;4:e6040.

14. Frierson Jr HF, El-Naggar AK, Welsh JB, et al. Large scale molecular analysis identifies genes with altered expression in salivary adenoid cystic carcinoma. Am J Pathol 2002;161:1315-1323.

15. Rooney DE, Czepulkowski BH. Malignancy and Acquired Abnormalities Human Cytogenetics: A Practical Approach, Vol. II, 2nd edn. Oxford University Press: New York, 1992.

16. Shaffer L, Slovak M, Campbell L. An International System for Human Cytogenetics Nomenclature. S. Karger: Basel, 2009.

17. Edwards PC, Bhuiya T, Kelsch RD. Assessment of p63 expression in the salivary gland neoplasms adenoid cystic carcinoma, polymorphous low-grade adenocarcinoma, and basal cell and canalicular adenomas. Oral Surg Oral Med Oral Pathol Oral Radiol Endod 2004;97: 613-619.

18. Emanuel $\mathrm{P}$, Wang $\mathrm{B}, \mathrm{Wu} \mathrm{M}$, et al. p63 Immunohistochemistry in the distinction of adenoid cystic carcinoma from basaloid squamous cell carcinoma. Mod Pathol 2005:18:645-650.

19. Conley J, Dingman DL. Adenoid cystic carcinoma in the head and neck (cylindroma). Arch Otolaryngol 1974;100:81-90.

20. West RB, Kong C, Clarke N, et al. MYB expression and translocation in adenoid cystic carcinomas and other salivary gland tumors with clinicopathologic correlation. Am J Surg Pathol 2011;35:92-99.

21. Kelland LR. Of mice and men: values and liabilities of the athymic nude mouse model in anticancer drug development. Eur J Cancer 2004;40:827-836.

22. Johnson Jl, Decker S, Zaharevitz D, et al. Relationships between drug activity in $\mathrm{NCl}$ preclinical in vitro and in vivo models and early clinical trials. Br J Cancer 2001;84:1424-1431.

23. Dong X, Guan J, English JC, et al. Patient-derived first generation xenografts of non-small cell lung cancers: promising tools for predicting drug responses for personalized chemotherapy. Clin Cancer Res 2010;16:1442-1451.

24. Fichtner I, Slisow W, Gill J, et al. Anticancer drug response and expression of molecular markers in early-passage xenotransplanted colon carcinomas. Eur J Cancer 2004;40:298-307. 